\title{
Clusters and higher moments of proton number fluctuations
}

\author{
Boris Tomášik* \\ Univerzita Mateja Bela, Banská Bystrica, Tajovského 40, Slovakia \\ FNSPE, České vysoké učení technické v Praze, Břehová 7, Prague 1, Czech Republic \\ E-mail: boris.tomasikecern.ch
}

\section{Zuzana Paulínyová}

Univerzita Pavla Jozefa Šafárika, Jesenná 5, Košice, Slovakia

\section{Jan Steinheimer}

Frankfurt Institute for Advanced Studies, Johann-Wolfgang-Goethe-Universität, Ruth-Moufgang-Straße 1, Frankfurt, Germany

\section{Marcus Bleicher}

Frankfurt Institute for Advanced Studies, Johann-Wolfgang-Goethe-Universität, Ruth-Moufgang-Straße 1,Frankfurt, Germany

Institut für theoretische Physik, Goethe-Universität Frankfurt, Max-von-Laue-Straße 1,

Frankfurt, Germany

GSI Helmholtzzentrum für Schwerionenforschung GmbH, Planckstraße 1, Darmstadt, Germany

Production of deuterons by coalescence has an important influence on the moments of the observed proton number distribution. Therefore, this effect must be taken into account when physics conclusions about baryon number fluctuation are drawn from the measurement of proton number fluctuations. We also show that a measurement of the third and fourth moments of the deuteron number distribution would allow to distinguish whether deuterons are produced statistically or by coalescence.

Critical Point and Onset of Deconfinement - CPOD2017

7-11 August, 2017

The Wang Center, Stony Brook University, Stony Brook, NY

\footnotetext{
${ }^{*}$ Speaker.
} 


\section{Introduction}

Moments of the proton number distribution are measured in ultrarelativistic nuclear collisions up to fourth order. The prime reason is the study of the QCD phase diagram, because they are used as a proxy for the higher order baryon number susceptibilities. The purpose of this paper is twofold. Firstly, we investigate how the skewness and kurtosis of the proton number distribution are influenced by coalescence which takes some of the protons and puts them into deuterons. Secondly, we point out that the measurement of the higher moments of deuteron number distribution can help to resolve the mechanism of deuteron production, whether it is coalescence or direct statistical production.

Higher order susceptibilities with respect to baryon number are being calculated on the lattice with increasing accuracy, as we have also witnessed at this conference [1,2]. They can be used for a more precise mapping of the QCD phase diagram because their values are very sensitive to the vicinity of the crossover from the confined to the deconfined state. For practical measurement they are related to the higher moments of the (net) baryon number distribution. Unfortunately, baryon number is actually not measurable at least because one cannot detect and count neutrons (and antineutrons). Therefore, the measurement must be based on protons only. Arguments exist that the proton number measurement is a good proxy for the full measurements of the baryon number $[3,4]$. However, the measured results for the moments of the proton number distribution may be influenced by other effects. Among them there are the conservation of baryon number, final state hadronic interactions [5], as well as issues like detector efficiency and acceptance limitations. It is important to realise that by going to as high as third and fourth moments of a number distribution the measurement becomes sensitive to rather fine details of the distribution.

Production of deuterons is among the processes which can affect the observed number of protons. With the binding energy of $2.2 \mathrm{MeV}$ a deuteron is too fragile to survive in an environment with the temperature of more than $100 \mathrm{MeV}$. Thus it is widely believed that the deuterons are made by coalescence of protons and neutrons. Their average number is usually well reproduced if one assumes that it is given as $B n_{p}^{2}$, where $n_{p}$ is the number of protons and $B$ is the coalescence factor. Here we will push the idea of proportionality further and assume that in each event the number of deuterons is proportional (up to fluctuations) to the actual number of protons to the second power. Subtracting in each event the protons which disappear in deuterons from the number of all protons will modify the number distribution of observed protons. At low energy deuteron production is a non-negligible effect. However, even if this effect becomes small at higher energies, it still could show up in the higher moments of proton distribution. We have formulated a model in this spirit and calculated the expected influence on the skewness and kurtosis of the proton number distribution.

Coming back to the mechanism of deuteron production, higher moments of their number distribution could help resolve the question how the deuterons are actually produced. Although the coalescence scenario seems quite natural, the mean yields can also be interpreted in terms of the statistical model. Clear difference between the two mechanisms of deuteron production appears in higher moments of deuteron number distribution. Statistical model should lead to Poissonian number distribution while coalescence couples the deuteron number distribution with that of protons (and neutrons). Once we have formulated the model of how this coupling is actually realised it is straightforward to use it not only for proton number fluctuations but also for the description of 
deuteron number fluctuations. We shall see the differences between results of the two models.

\section{The observables}

In this paper we will evaluate moments of the proton number distributions. In the second part we shall also study the distribution of the deuteron number $n_{d}$. Averages over events are denoted with angular brackets, so that $\left\langle n_{p}\right\rangle$ and $\left\langle n_{d}\right\rangle$ stand for the mean proton number and mean deuteron number, respectively.

We shall look at the variance

$$
\sigma^{2}=\left\langle\left(n_{p}-\left\langle n_{p}\right\rangle\right)^{2}\right\rangle
$$

the skewness

and the kurtosis

$$
S=\frac{\left\langle\left(n_{p}-\left\langle n_{p}\right\rangle\right)^{2}\right\rangle}{\sigma^{3}}
$$

$$
\kappa=\frac{\left\langle\left(n_{p}-\left\langle n_{p}\right\rangle\right)^{2}\right\rangle}{\sigma^{4}}-3
$$

of the number distributions.

\section{The model}

We formulate here the simplest model which takes into account that deuteron number scales with the square of the proton number.

We start by assuming that the number of all initial protons $n_{i}$ fluctuates according to a Poisson distribution with the mean $\lambda_{p}$

$$
P_{i}\left(n_{i}\right)=\lambda_{p}^{n_{i}} \frac{e^{-\lambda_{p}}}{n_{i} !}
$$

It is important to realise that $n_{i}$ is not measurable, since it also includes those protons which become parts of deuterons. Thus also $\lambda_{p}$ is not directly observable and will have to be obtained from common analysis of proton and deuteron yields.

Production of deuterons is random process, as well. We assume that the actual number of deuterons in every event is drawn from a Poisson distribution. The mean of that distribution in an event with $n_{i}$ initial protons is

$$
\lambda_{d}=B n_{i}^{2}
$$

as dictated by the coalescence model. The coalescence parameter $B$ is to be determined from experimental data. Then, the conditional probability to have $n_{d}$ deuterons in an event with $n_{i}$ initial protons is

$$
P_{d}\left(n_{d} \mid n_{i}\right)=\lambda_{d}^{n_{d}} \frac{e^{-\lambda_{d}}}{n_{d} !}=\left(B n_{i}^{2}\right)^{n_{d}} \frac{e^{-B n_{i}^{2}}}{n_{d} !} .
$$

Combining this distribution with that of initial protons given by eq. (3.1) we can obtain the distribution for the number of observed protons $n_{p}$. We recall that those protons are observed which do not disappear from the balance as parts of deuterons. Thus

$$
n_{p}=n_{i}-n_{d}
$$


and the corresponding distribution is obtained as

$$
P\left(n_{p}\right)=\sum_{n_{i} \geq n_{p}} P_{i}\left(n_{i}\right) P_{d}\left(n_{i}-n_{p} \mid n_{i}\right) .
$$

The sum on the right-hand side can be evaluated and thus we know the whole distribution. From this all moments can be calculated via equations (2.1-2.3).

In order to obtain the observed number of deuterons we have to sum the conditional probability $P_{d}\left(n_{d} \mid n_{i}\right)$ for all possible values of $n_{i}$. Thus we arrive at the folding

$$
P_{d}\left(n_{d}\right)=\sum_{n_{i} \geq n_{d}} P_{d}\left(n_{d} \mid n_{i}\right) P_{i}\left(n_{i}\right) .
$$

This distribution can be evaluated straightforwardly. Again, all its moments can be calculated, as well.

In summary, we have two parameters in our model: the mean initial number of protons $\lambda_{p}$ and the coalescence parameter $B$. They have to be determined separately for every collision energy. The two observables that are used to determine them are the mean observed proton number and the mean deuteron number

$$
\begin{aligned}
\left\langle n_{p}\right\rangle & =\sum_{n_{p}} n_{p} P_{p}\left(n_{p}\right) \\
\left\langle n_{d}\right\rangle & =\sum_{n_{d}} n_{d} P_{d}\left(n_{d}\right) .
\end{aligned}
$$

In order to get the deuteron number at all energies that we need, we have fitted the collision energy dependence of the deuteron-to-proton ratio

$$
\frac{\left\langle n_{d}\right\rangle}{\left\langle n_{p}\right\rangle}=0.8\left[\frac{\sqrt{s_{N N}}}{1 \mathrm{GeV}}\right]^{-1.55}+0.0036 .
$$

The quality of the fit can be inspected from Fig. 1.

Using these formulas we have calculated the higher moments of the proton number distribution. At higher collision energies, antiprotons also have to be taken into account. In principle, they can also make the antideuterons and this is expressed via the same type of distributions as we have had for protons and deuterons. In this case, however, we made Monte Carlo simulations of proton and antiproton production according to the formulated model. At lower energies we have checked that the MC simulation and the direct calculation lead to the same results.

\section{Results for net proton number fluctuations}

With the help of the derived model we have determined the values of the scaled skewness $S \sigma$ and scaled kurtosis $\kappa \sigma^{2}$ as functions of the mean observed proton number $\left\langle n_{p}\right\rangle$ and the coalescence parameter $B$. The results are plotted in Fig. 2. First of all we see that if no deuterons are produced, i.e. at $B=0$, we recover the trivial values for the Poisson distribution: $S \sigma=\kappa \sigma^{2}=1$. On the other hand, many deuterons are produced if the number of protons is large or for large value of the coalescence parameter $B$. Then the scaled moments are modified dramatically. In most extreme 


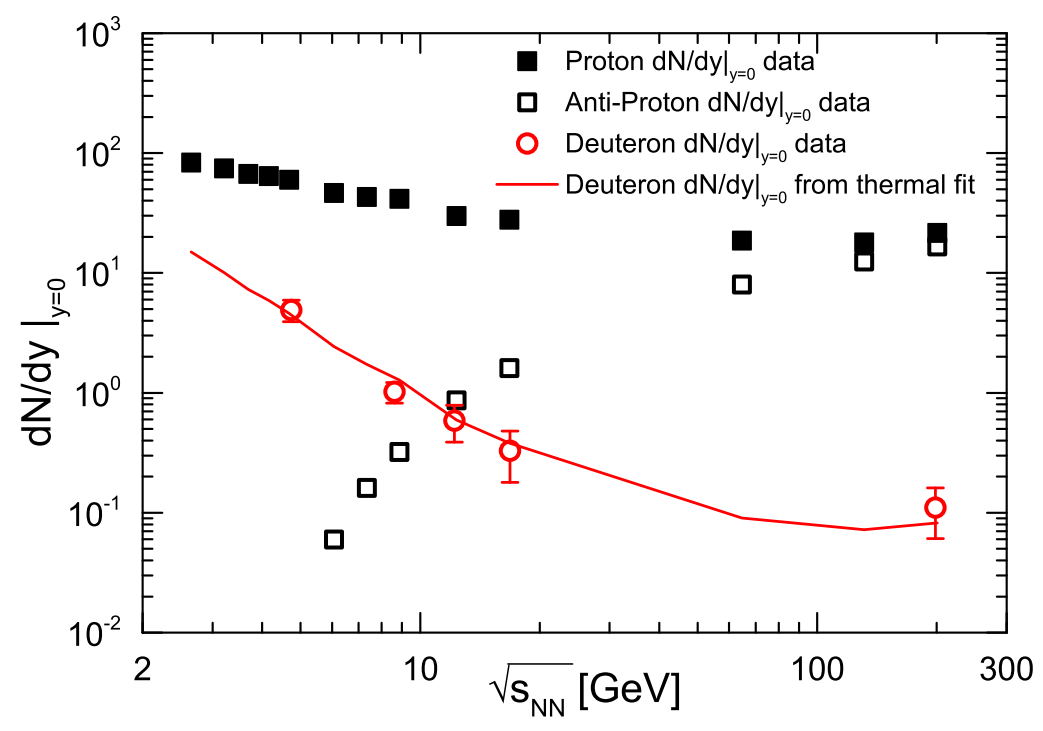

Figure 1: Fit to the deuteron yields at midrapidity based on the parametrisation (3.9). Experimental data from $\mathrm{Au}+\mathrm{Au}$ collisions are plotted for protons, antiprotons [6], and deuterons [7].
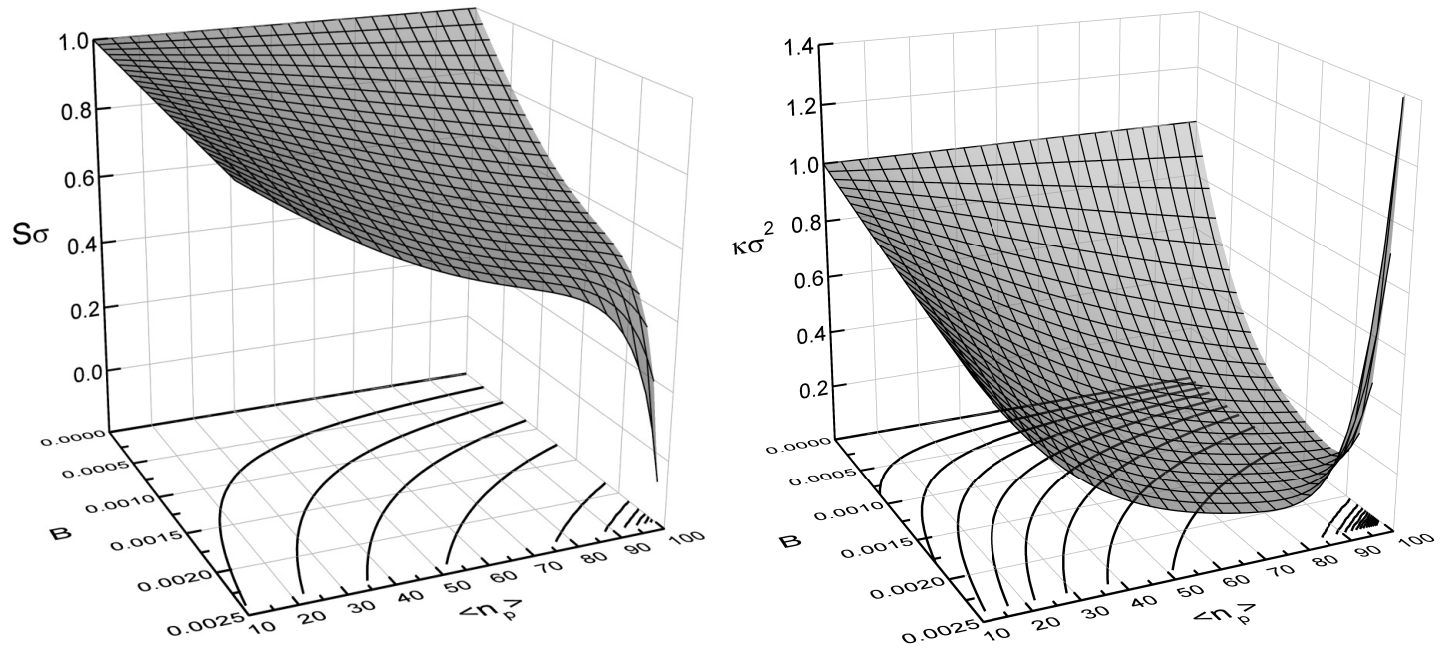

Figure 2: Scaled skewness $S \sigma$ (left) and scaled kurtosis $\kappa \sigma^{2}$ (right) as functions of mean observed proton number $\left\langle n_{p}\right\rangle$ and the coalescence parameter $B$.

cases they go down as low as to 0.2 . The values of $\left\langle n_{p}\right\rangle$ and $B$ in that figure are realistic and the highest values can be attained in nuclear collisions at lower BNL-AGS energies. We thus stress that the effect of the deuteron production on the shape of the proton number distribution may play an important role and definitely must be taken into account.

In order to better understand where the large modification of the values comes from, we have also plotted the proton and deuteron number distributions (Fig. 3). They have been calculated for values of $\left\langle n_{p}\right\rangle$ and $B$ relevant for central $\mathrm{Au}+\mathrm{Au}$ collisions at $\sqrt{s_{N N}}=2.6 \mathrm{GeV}$. In general, 

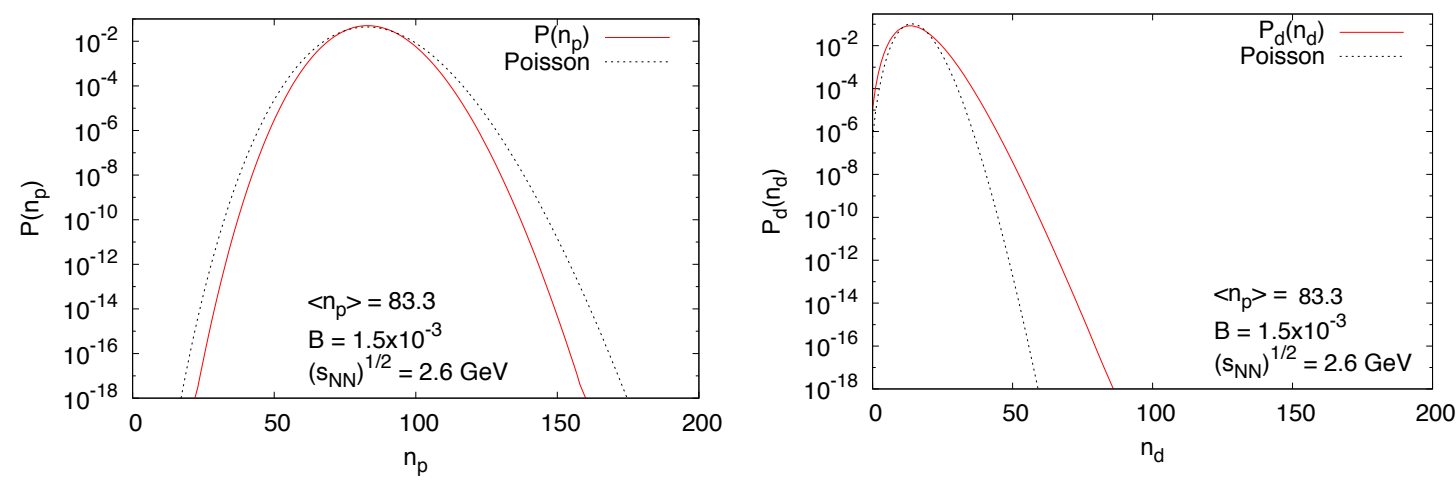

Figure 3: Number distribution of observed protons (left) and of deuterons (right) calculated for $\left\langle n_{p}\right\rangle=83.4$ and $B=1.6 \times 10^{-3}$. These parameter values describe proton and deuteron production from $\mathrm{Au}+\mathrm{Au}$ collisions at $\sqrt{s_{N N}}=2.6 \mathrm{GeV}$. For comparison, dashed curves show the Poisson distribution with the same mean value as the calculated distributions.

we observe that for the proton number distribution, production of deuterons cuts from the highmultiplicity end because there coalescence is more pronounced, since it is proportional to the square of the proton number. Hence, in general, the observed proton number distribution is narrower than the Poisson. The overall effect is that the resulting $P_{p}\left(n_{p}\right)$ has smaller tails and is more skewed towards lower proton numbers than the Poisson distribution. This is quantified by the calculated skewness and kurtosis.

On the obtained deuteron distribution the effect is just opposite. Since the mean deuteron number is proportional to $n_{i}^{2}$, the high multiplicity tail of the distribution is enhanced and really heavy. It is clearly heavier than the reference Poisson distribution. This will result in significantly larger values for the skewness and kurtosis later in this paper.

After we have understood the results qualitatively, we are ready to plug in phenomenologically relevant values of the parameters and calculate the collision energy dependence of the skewness and kurtosis. Recall that we want to study the fluctuations of the net proton number. However, at energies below $\sqrt{s_{N N}} \approx 10 \mathrm{GeV}$ antiproton production can be neglected and we can just look at proton number distribution. In this interval we have calculated the higher moments with the formulas from Section 3. As a cross-check, $S \sigma$ and $\kappa \sigma^{2}$ have been determined by Monte Carlo simulations for all collision energies. The results are plotted in Fig. 4.

When also antiprotons are produced, Skellam distribution is expected if both proton and antiproton numbers follow the Poisson distribution. Therefore, for the skewness we also plot the ratio of our result to the expectation due to Skellam distribution. This is important. Our results show a dramatic decrease of $S \sigma$ as the collision energy increases to top RHIC energies of 130 and $200 \mathrm{GeV}$ pre nucleon pair. However, when divided by the value due to Skellam distribution we see that the ratio converges towards 1 , so that the result is trivial. Nevertheless, we see that the results for collision energies below $10 \mathrm{GeV}$ per nucleon pair are strongly influenced by formation of deuterons. 

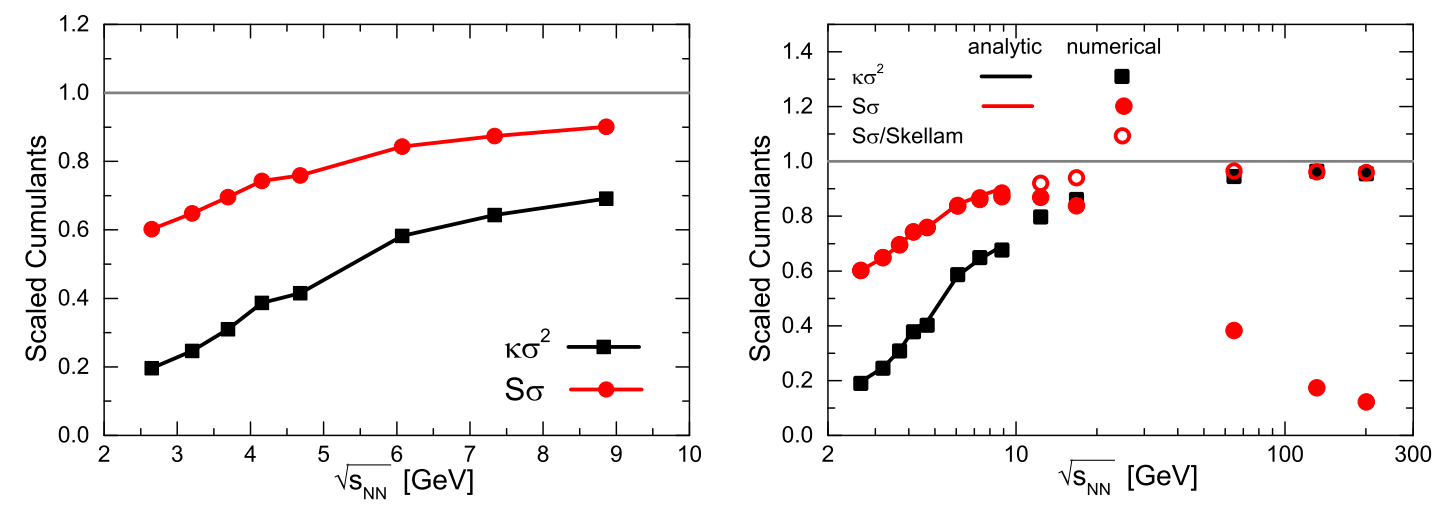

Figure 4: Scaled skewness $S \sigma$ and scaled kurtosis $\kappa \sigma^{2}$ as functions of collision energy for central Au+Au collisions. In the left panel no antiprotons are taken into account and results are calculated with the help of formulas from Section 3. In the right panel antiproton production has been taken into account and results are obtained from Monte Carlo simulations.

\section{Thermal production vs coalescence of deuterons}

If deuterons are produced simply according to the statistical model, then their number distribution should be Poissonian and $S \sigma=\kappa \sigma^{2}=1$. As it was illustrated in Fig. 3 right, coalescence leads to skewness and kurtosis which are much increased with respect to that expectation. However, we note that this result was based on rather strong assumption that the mean number of deuterons scales with $n_{i}^{2}$. As a matter of fact, deuterons are bound states of a proton and a neutron and so it would be more natural to expect that

$$
\lambda_{d}=B n_{i} n_{j}
$$

where $n_{j}$ is the initial number of all neutrons, analogically to initial number of all protons $n_{i}$. The number of neutrons fluctuates according to Poisson distribution for which we assume the same mean value as we did for initial proton number distribution. The conditional probability that $n_{d}$ deutrons are observed in case where $n_{i}$ initial protons and $n_{j}$ initial neutrons were produced is

$$
P\left(n_{d} \mid n_{i}, n_{j}\right)=\lambda_{d}^{n_{d}} \frac{e^{-\lambda_{d}}}{n_{d} !}=\left(B n_{i} n_{j}\right)^{n_{d}} \frac{e^{-B n_{i} n_{j}}}{n_{d} !} .
$$

Consequently, the distribution of deuteron number is

$$
P_{d}\left(n_{d}\right)=\sum_{n_{i}, n_{j} \geq n_{d}} P_{d}\left(n_{d} \mid n_{i}, n_{j}\right) P_{i}\left(n_{i}\right) P_{j}\left(n_{j}\right)
$$

For later reference, we call the two models that we have formulated here as

Model A given by eqs. (3.2), (3.3), and (3.6);

Model B given by eqs. (5.1), (5.2), and (5.3). 


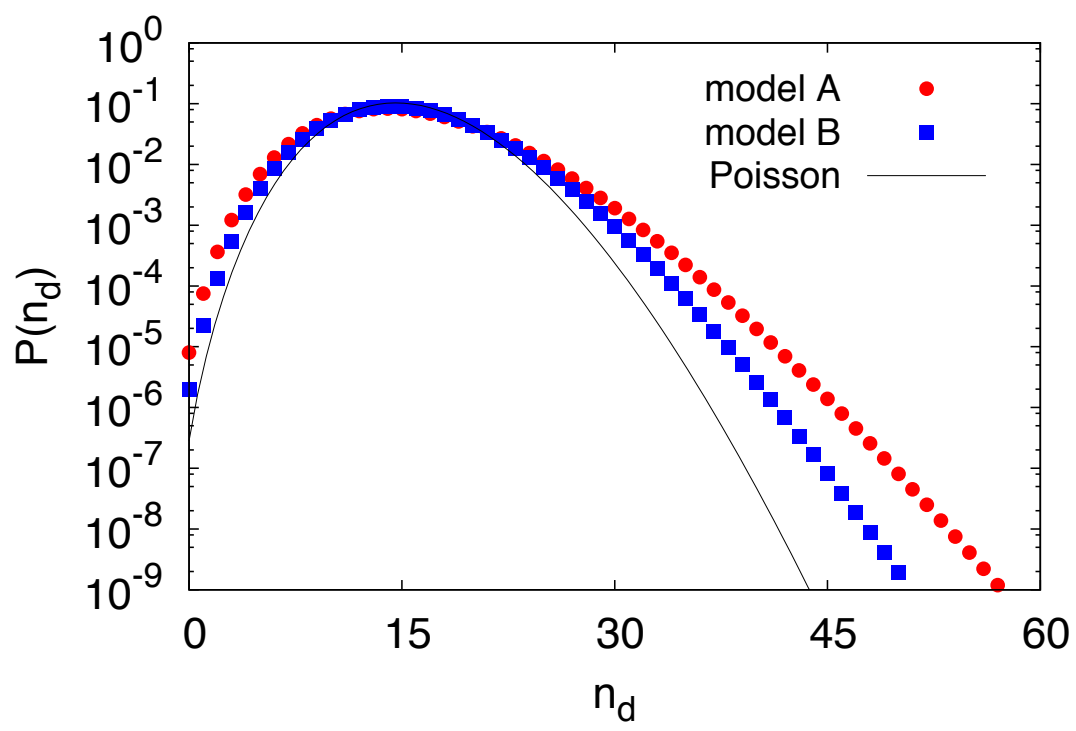

Figure 5: Deuteron number distributions determined for model parameters which correspond to $\mathrm{Au}+\mathrm{Au}$ collisions at $\sqrt{s_{N N}}=2.6 \mathrm{GeV}$. All distributions have the same mean.

\section{Results for deuteron number fluctuations}

We first inspect the difference between the individual models in Fig. 5. It shows the deuteron number distributions with parameters suitable for the description of proton and deuteron production in $\mathrm{Au}+\mathrm{Au}$ collisions at $\sqrt{s_{N N}}=2.6 \mathrm{GeV}$. It is seen that both Model A and Model B lead to number distributions which are clearly more skewed and have heavier tails than the reference with the same mean which is provided by the Poisson distribution. While Poisson distribution leads to $\sigma^{2} /\left\langle n_{d}\right\rangle=S \sigma=\kappa \sigma^{2}=1$, we obtain for Model A: $\sigma^{2} /\left\langle n_{d}\right\rangle=1.609, S \sigma=2.218, \kappa \sigma^{2}=6.915$; and for Model B: $\sigma^{2} /\left\langle n_{d}\right\rangle=1.308, S \sigma=1.616, \kappa \sigma^{2} 2=3.422$.

Encouraged by this result we can now determine $P_{d}\left(n_{d}\right)$ for all collision energies and calculate the higher moments. The results are plotted in Fig. 6 for Model A (left) and Model B (right). In general, we can summarise that going to lower energies makes the effect of coalescence more visible. It is also more visible in the higher moments of the distribution. The difference with respect to Poissonian result is visible in case of both Model A a Model B, although it is about twice as big for Model A than it is for Model B. We thus offer a good motivation for a measurement of higher moments of deuteron distribution.

\section{Conclusions}

Proton number fluctuations are nowadays being widely studied because of their connection to baryon number fluctuations.

Here we have shown that the production of deuterons must be taken into account if any physics conclusions are to be drawn from the measurement. The disappearance of some protons into deuterons has an important effect on the proton number distribution [8]. 

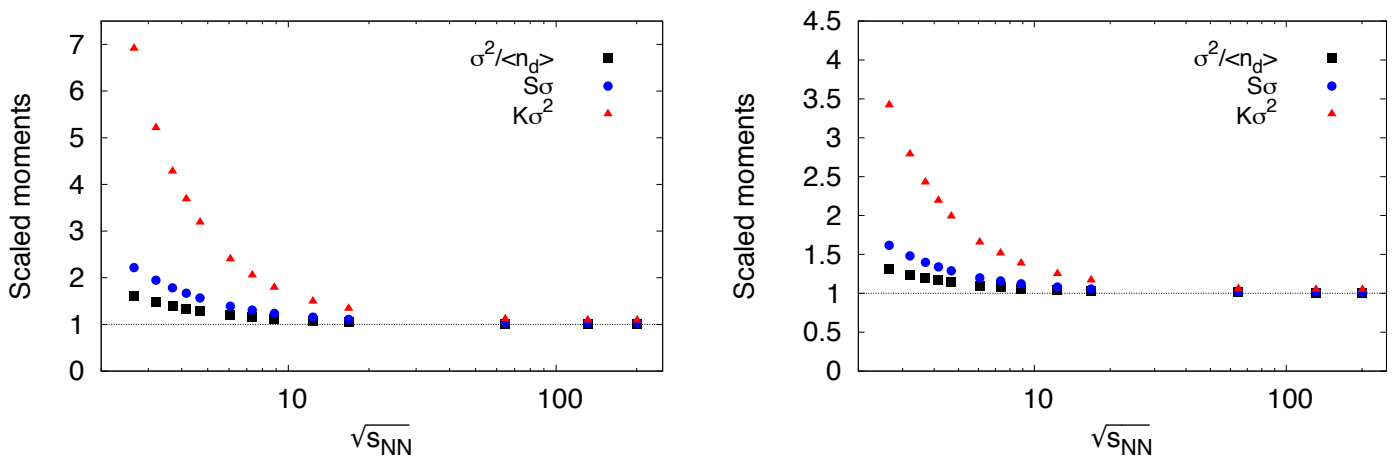

Figure 6: Collision energy dependence for $\sigma^{2} /\left\langle n_{d}\right\rangle, S \sigma$, and $\kappa \sigma^{2}$ for the model with deuteron production depending on proton number squared (Model A, left) and the model assuming independent proton and neutron number fluctuations (Model b, right).

We have also shown that by studying higher moments of the deuteron number distribution one will be able to decide if the deuterons are produced according to the statistical model or if their production is rather described by coalescence [9].

Acknowledgments We acknowledge the support by VEGA 1/0469/15 (Slovakia). The work has been supported by the grant 17-04505S of the Czech Science Foundation (GAČR). The collaboration has been facilitated by the DAAD PPP project and by the COST Action CA15213 THOR.

\section{References}

[1] A. Bazavov et al. [HotQCD Collaboration], Phys. Rev. D 96 (2017) no.7, 074510 doi:10.1103/PhysRevD.96.074510 [arXiv:1708.04897 [hep-lat]].

[2] J. Günther, these proceedings.

[3] M. Kitazawa and M. Asakawa, Phys. Rev. C 85 (2012) 021901 doi:10.1103/PhysRevC.85.021901 [arXiv:1107.2755 [nucl-th]].

[4] M. Kitazawa and M. Asakawa, Phys. Rev. C 86 (2012) 024904 Erratum: [Phys. Rev. C 86 (2012) 069902] doi:10.1103/PhysRevC.86.024904, 10.1103/PhysRevC.86.069902 [arXiv:1205.3292 [nucl-th]].

[5] J. Steinheimer, V. Vovchenko, J. Aichelin, M. Bleicher and H. Stöcker, Phys. Lett. B 776 (2018) 32 doi:10.1016/j.physletb.2017.11.012 [arXiv:1608.03737 [nucl-th]].

[6] A. Andronic, P. Braun-Munzinger and J. Stachel, Nucl. Phys. A 772 (2006) 167 doi:10.1016/j.nuclphysa.2006.03.012 [nucl-th/0511071].

[7] A. Andronic, P. Braun-Munzinger, J. Stachel and H. Stöcker, Phys. Lett. B 697 (2011) 203 doi:10.1016/j.physletb.2011.01.053 [arXiv:1010.2995 [nucl-th]].

[8] Z. Fecková, J. Steinheimer, B. Tomášik, M. Bleicher, Phys. Rev. C 92 (2015) 064908.

[9] Z. Fecková, J. Steinheimer, B. Tomášik, M. Bleicher, Phys. Rev. C 93 (2016) 054906. 